\title{
Inlet effects on roll-wave development in shallow turbulent open-channel flows
}

\author{
Francesca Campomaggiore ${ }^{1}$, Cristiana Di Cristo ${ }^{2}$, Michele Iervolino $^{3}$, Andrea Vacca $^{3}$ \\ ${ }^{1}$ Dipartimento di Ingegneria e Architettura, Università di Trieste, Piazzale Europa 1, 34127 Trieste, Italy. \\ 2 Dipartimento di Ingegneria Civile e Meccanica, Università di Cassino e del Lazio Meridionale, Via Di Biasio 43, 03043 Cassino (FR), \\ Italy. \\ ${ }^{3}$ Dipartimento di Ingegneria Civile, Design, Edilizia ed Ambiente, Seconda Università di Napoli, Via Roma 29, 81031 Aversa (CE), Italy. \\ * Corresponding author. Tel.: +39 081 5010344. Fax: + 39081 5037370. E-mail: vacca@unina.it
}

\begin{abstract}
The present work investigates the effect of the flow profile induced by an inlet condition on the roll-wave evolution in turbulent clear-water flows. The study employs theoretical and numerical analyses. Firstly, the influence of the inlet condition on the spatial evolution of a single perturbation in a hypercritical flow is examined through the expansion near a wavefront analysis. The results show that an accelerated unperturbed profile reduces the disturbance spatial growth. A decelerated profile causes an increase. The effect of the flow profile on the spatial evolution of rollwave trains is then numerically investigated solving the Saint Venant equations with a second-order Runge-Kutta Total Variation Diminishing (TVD) Finite Volume scheme. The numerical simulations comply with the analytical results for the initial and transition phases of the roll-wave development. The unperturbed profile influences even the roll-waves statistical characteristics in the final stage, with a more evident effect in case of accelerated profiles. The influence of the flow profile should be therefore accounted for in the formulation of predictive criteria for roll-waves appearance based on the estimation of the disturbance spatial growth rate.
\end{abstract}

Keywords: Open-channel flows; Roll-waves; Hypercritial flows; Numerical simulation.

\section{INTRODUCTION}

Water flowing down an inclined solid surface may exhibit a surface instability, appearing as downstream propagating wave trains. Similar waves, usually named roll-waves, occur in both torrential rivers and manmade steep-sloped channels (Chanson 2004; Kenyon, 1998). They also affect overland flows, such as rill flows (Kranenburg, 1992; Liu et al., 2005) and concern also non-Newtonian fluids, such as laminar mud-flows (Liu and Mei, 1989). Roll-waves may cause overflows and intermittent forces at the outlet with the risk of infrastructure damages; they may affect the sediment transport in rivers, increasing the erosion capacity. The prediction of roll-wave occurrence is of great interest for the environmental engineering. This phenomenon has been deeply studied, through both theoretical investigations and experimental observations indicating that the roll-waves arise from inertial instability (Brock, 1969; Logan and Iverson, 2007).

Within the shallow-water approximation, the Saint Venant equations (SVEs), either in their hyperbolic or diffusive formulation, represent the main framework for the theoretical studies of roll-waves in turbulent flows. Assuming a uniform unperturbed condition, different theoretical approaches, with increasing degrees of complexity, have been applied for studying the occurrence and the development of roll-waves.

Linear stability analyses are able to describe the growth of an infinitesimal disturbance only in the first phase of the instability process. In such a framework, the hyperbolic formulation of SVEs has been the most used one. Owing to the convective character of the instability (Di Cristo and Vacca, 2005; Di Cristo et al., 2012a; Ridolfi et al., 2006; Thual et al., 2010) both temporal and spatial analyses have been performed. Temporal analysis led to individuate the limiting values of the relevant dimensionless parameters for the stability (e.g. Berlamont, 1976; Berlamont and Vanderstappen, 1981; Brock, 1970;
Di Cristo et al., 2012b; Ponce and Simon, 1977; Venutelli, 2011). The spatial growth of the disturbance in the initial phase has been evaluated through the spatial linear analysis with either a pointwise (e.g. Liggett, 1975) or an oscillating (Di Cristo et al., 2010; Supino, 1960; Thual et al., 2010) perturbation. Based on the spatial linear analysis results, minimum channel length criteria have been proposed for engineering purposes considering both Chezy (Di Cristo et al., 2008; Liggett, 1975; Montuori, 1963) and Colebrook-White (Di Cristo et al., 2010) resistance formulas.

Weakly non-linear analyses allow describing the spatial evolution of a small (but finite) disturbance. Kranenburg (1992) considered a diffusive formulation of SVEs with a constant turbulent viscosity along with the Chezy formula, showing that periodic roll-waves are unstable to a subharmonic disturbance. The growth of this disturbance destroys the instabilities within a finite interval through the mechanism of shock coalescence, developing larger roll-waves. Kevorkian et al. (1995), using instead the hyperbolic SVEs with the Chezy formula, determined the conditions under which periodic linearly unstable disturbances evolve into travelling waves consisting of piecewise continuous profiles joined by shocks. Balmforth and Mandre (2004) explored the effect of bottom topography through both linear stability analysis and asymptotic expansion with multiple time and length scales. The authors assumed the diffusive model, with a constant turbulent viscosity, and a Chezy parameterization. Low-amplitude topography has been found to destabilize turbulent flows, with a lower minimum threshold value of the Froude number required for instability. The trend reverses with higher topographic amplitude, which stabilizes the flow.

The entire roll-waves development process may be described only through the fully nonlinear simulations of SVEs. Zanuttigh and Lamberti (2002), considering the hyperbolic version of SVEs with the Colebrook-White formula, have shown that this 
model is able to describe all the essential features of the rollwaves. Balmforth and Mandre (2004) used diffusive SVEs with the Chezy formula and a turbulent viscosity term with a constant viscosity parameter. The authors numerically investigated the effect of bottom topography on the stability of a uniform turbulent flow, finding that roll-wave trains undergo coarsening and merging due to waves overtaking and lengthening the pattern scale. Huang and Lee (2015) numerically solved the diffusive SVEs, with a constant turbulent viscosity value and considering the Colebrook-White formula for the bottom shear stress. Comparing their results with experimental data of Brock (1967), the authors concluded that the inclusion of the diffusive term in SVEs allowed an improvement of the quantitative agreement with the experimental findings. However, the results quality was strongly dependent on the turbulent viscosity value, which indeed had to be calibrated. Cao et al. (2015) proposed a physically-enhanced shallow-water hydrodynamic model, which explicitly incorporates turbulent Reynolds stress through a $k$ - $\varepsilon$ model, along with a Chezy formula. The Authors applied their model to simulate the experiments by Brock (1967), reproducing the final pattern of both periodic and natural rollwaves. The comparison of their numerical results with those by Zanuttigh and Lamberti (2002) indicates that the increased model complexity appreciably improved only the prediction of the mean water depth at trough. Small differences between the results of the two models were observed for the mean water depth at crest and for the mean wave period.

All the aforementioned studies address the development of roll-waves starting from uniform unperturbed flow.

However, in many engineering applications involving steep slope channels, gradually varied hypercritical profiles are encountered. For instance, as far as spillways are concerned, depending either on the type of the inlet (e.g. overflow, orifice, etc...) or on the control device (e.g. unregulated, sluice-gate, etc...), accelerated or decelerated hypercritical profiles are very often generated (Khatsuria, 2004).

The roll-waves occurrence in presence of non-uniform flow conditions has been so far analyzed only from the stability point of view. Stability limits of several gradually varied hypocritical and hypercritical profiles have been found by Dracos and Glenne (1967), considering the hyperbolic SVEs along with the Chezy formula. The authors, studying the spatial evolution of a discontinuity on the free surface, i.e. using the expansion near a wavefront technique (Witham, 1974), demonstrated a strong influence of the local Froude number on the stability of the unperturbed profile. The results of Dracos and Glenne (1967) have been confirmed by Kranenburg (1990), through a weakly non-linear analysis of the same flow model. Although some examples of the evolution of the wave amplitude with distance have been discussed in Kranenburg (1990), a thorough study concerning the influence of unperturbed hypercritical profiles on the perturbation spatial evolution is not available, not even within the linear approximation.

From the above literature review, it follows that differently from the case of uniform condition, the spatial evolution of the roll-waves in presence of a gradually varied profile has been only marginally investigated.

Based on this motivation, the present paper focuses on the roll-wave development in presence of both accelerated and decelerated hypercritical profiles in steep channels. The hyperbolic version of the SVEs, which is able to capture the essential features of the roll-waves, is considered as the flow model. Colebrook-White formula is employed to model the friction factor. The study develops analytical and numerical analyses. As far as the former approach is concerned, the expansion near a wavefront technique (Witham, 1974) is firstly applied to generalize theoretical achievements of Dracos and Glenne (1967), accounting for the resistance coefficient variability. Therefore, the spatial evolution of a single perturbation in linear and non-linear regime, up to the occurrence of wave breaking, is analyzed. Then, the development of natural roll-wave trains is studied considering unperturbed accelerated and decelerated profiles through numerical simulations of the SVEs, discretized with a second-order Runge-Kutta Total Variation Diminishing (TVD) Finite Volume Method.

The paper is structured as follows. In section 2 the dimensional and dimensionless governing equations are given. In Section 3 the extension of the expansion near a wavefront technique considering the friction coefficient variability is provided. A description of the numerical method used for simulating the effect of the gradually varied hypercritical profiles is given in Section 4. The spatial evolution of a single perturbation and the natural roll-waves train development are both reported and discussed in Section 5. Finally, the conclusions are provided in Section 6.

\section{GOVERNING EQUATIONS}

Let us consider a homogeneous and incompressible fluid flowing in an infinitely wide channel inclined at angle $\theta$ with respect to the horizontal plane, without lateral inflow or outflow. Under the hypothesis of negligible vertical acceleration and assuming spatial variations to occur over scales larger than flow depth, the dimensional depth-averaged momentum and mass conservation equations (Saint Venant Equations) in their hyperbolic formulation are:

$$
\frac{\partial \tilde{h}}{\partial \tilde{t}}+\frac{\partial \tilde{u} \tilde{h}}{\partial \tilde{x}}=0
$$

$$
\frac{\partial(\tilde{h} \tilde{u})}{\partial \tilde{t}}+\frac{\partial}{\partial \tilde{x}}\left(\tilde{h} \tilde{u}^{2}\right)-g \tilde{h}\left(\sin \theta-\cos \theta \frac{\partial \tilde{h}}{\partial \tilde{x}}\right)+\frac{\tilde{\tau}_{b}}{\rho}=0
$$

in which $\tilde{h}$ is the flow depth, $\tilde{u}$ is the depth-integrated streamwise velocity component, $g$ is the acceleration due to gravity, $\rho$ is the water density, and finally $\tilde{x}$ and $\tilde{t}$ are the streamwise coordinate and time, respectively. The bottom stress $\tilde{\tau}_{b}$ is expressed by:

$$
\tilde{\tau}_{b}=\lambda \frac{\rho \tilde{u}^{2}}{8}
$$

where the resistance coefficient $\lambda$ is evaluated through the implicit Colebrook-White formula:

$$
\frac{1}{\sqrt{\lambda}}=a \ln \left(\frac{b}{\operatorname{Re} \sqrt{\lambda}}+d \frac{\tilde{\varepsilon}}{\tilde{h}}\right)
$$

in which $\operatorname{Re}=\tilde{u} \tilde{h} / v$ denotes the Reynolds number, $v$ is the kinematic viscosity and $\tilde{\varepsilon}$ is the dimensional sand equivalent roughness. In Eq. (4) the $a, b$, and $d$ coefficients are dimensionless experimental parameters.

Denoting with subscript $N$ the normal values, i.e. in uniform condition of flow, the following dimensionless variables are introduced: 
$h=\frac{\tilde{h}}{\tilde{h}_{N}}, x=\frac{\tilde{x}}{\tilde{l}_{N}}, t=\frac{\tilde{u}_{N}}{\tilde{l}_{N}} \tilde{t}, u=\frac{\tilde{u}}{\tilde{u}_{N}}$,

with $\tilde{l}_{N}=\tilde{h}_{N} \cot \theta$. The system (1)-(2) may be rewritten in its dimensionless form as follows:

$$
\begin{aligned}
& \frac{\partial h}{\partial t}+u \frac{\partial h}{\partial x}+h \frac{\partial u}{\partial x}=0 \\
& \frac{\partial u}{\partial t}+u \frac{\partial u}{\partial x}+\frac{1}{F_{N}^{2}} \frac{\partial h}{\partial x}=\frac{1}{F_{N}^{2}}\left(1-\frac{\lambda}{\lambda_{\mathrm{N}}} \frac{u^{2}}{h}\right)
\end{aligned}
$$

in which:

$$
\begin{aligned}
& \frac{1}{\sqrt{\lambda}}=a \ln \left(\frac{b}{u h e_{N} \sqrt{\lambda}}+d \frac{\varepsilon_{N}}{h}\right) \text { and } \\
& \frac{1}{\sqrt{\lambda_{N}}}=a \ln \left(\frac{b}{R e_{N} \sqrt{\lambda_{N}}}+d \varepsilon_{N}\right)
\end{aligned}
$$

where $\varepsilon_{N}=\tilde{\varepsilon} / \tilde{h}_{N}$ and

$$
\operatorname{Re}_{N}=\frac{\tilde{u}_{N} \tilde{h}_{N}}{v}, F_{N}=\frac{\tilde{u}_{N}}{\sqrt{g \tilde{h}_{N} \cos \theta}}
$$

denote the Reynolds and Froude numbers in the uniform condition, respectively.

\section{EXPANSION NEAR A WAVEFRONT WITH VARIABLE FRICTION COEFFICIENT}

The present section aims to extend the results of Dracos and Glenne (1967), pertaining to the Saint Venant equations with constant friction coefficient, accounting for the friction coefficient variability. To this aim the near wavefront expansion analysis (Witham, 1974), based on the perturbation of a gradually varied profile with a discontinuity in the first derivatives of the $h$ and $u$ variables, is performed. Such a technique, although it allows to analyze only the spatial evolution of a single perturbation, has the advantage to reveal the effect of a gradually varied unperturbed profile on the growth rate of the disturbance, through a simple ordinary differential equation. The analysis exploits the hyperbolic character of system (6)-(7) for which a discontinuity propagates on the characteristics lines in the $(t, x)$ plane and in particular on the fastest one. Near wavefront expansion technique has been recently employed to analyze the stability properties of hypocritical profiles in nonNewtonian fluids (Di Cristo et al., 2015) and, in its linearized version, to provide engineering criteria for roll-waves prediction in uniform condition of flow with either constant (Liggett, 1975) or variable (Di Cristo et al., 2010) friction coefficient. Denoting with $\mathbf{u}^{\mathrm{T}}=[h, u]$ the unknown vector, the Saint Venant equations may be rewritten in the following form:

$$
\frac{\partial \mathbf{u}}{\partial t}+\mathbf{A}(\mathbf{u}) \frac{\partial \mathbf{u}}{\partial x}=\mathbf{s}(\mathbf{u})
$$

in which
$\mathbf{A}=\left[\begin{array}{cc}u & h \\ \frac{1}{F_{N}^{2}} & u\end{array}\right], \mathbf{s}=\left[\begin{array}{c}0 \\ \frac{1}{F_{N}^{2}}\left(1-\frac{\lambda}{\lambda_{N}} \frac{u^{2}}{h}\right)\end{array}\right]$

The eigenvalues and the left eigenvectors of the matrix $\mathbf{A}$ are:

$$
\kappa^{ \pm}=u \pm \frac{\sqrt{h}}{F_{N}}, \mathbf{I}^{ \pm}=\left[\begin{array}{c}
1 \\
\pm F_{N} \sqrt{h}
\end{array}\right]
$$

Denoting with subscript 0 the quantities pertaining to the unperturbed condition, the following variables transformation is introduced:

$\xi=x, \quad d \tau=\kappa_{0}^{+}(\xi) d t-d x$

Accounting for Eq. (13), the system (10) may be rewritten in the $\xi$ and $\tau$ variables as follows:

$\kappa_{0}^{+}(\xi) \frac{\partial \mathbf{u}}{\partial \tau}+\mathbf{A}(\mathbf{u})\left(\frac{\partial \mathbf{u}}{\partial \xi}-\frac{\partial \mathbf{u}}{\partial \tau}\right)=\mathbf{s}(\mathbf{u})$

The unknown vector $\mathbf{u}$, the matrix $\mathbf{A}$ and the source term $\mathbf{s}$ are then expanded in series around $\tau=0$ (Witham, 1974):

$\mathbf{u}(\xi, \tau)=\mathbf{u}_{0}(\xi)+\mathbf{u}_{1}(\xi) \tau+\mathbf{u}_{2}(\xi) \tau^{2}+\ldots$

$\mathbf{A}(\xi, \tau)=\mathbf{A}_{0}(\xi)+\mathbf{A}_{1}(\xi) \tau+.$.

$\mathbf{s}(\xi, \tau)=\mathbf{s}_{0}(\xi)+\mathbf{s}_{1}(\xi) \tau+\ldots$

Omitting for simplicity the dependency of flow variables on $\xi$, the following definitions hold:

$\mathbf{A}_{0}=\left[\begin{array}{cc}u_{0} & h_{0} \\ \frac{1}{F_{N}^{2}} & u_{0}\end{array}\right], \mathbf{A}_{1}=\left[\begin{array}{cc}u_{1} & h_{1} \\ 0 & u_{1}\end{array}\right]$

$\mathbf{s}_{0}=\left[\begin{array}{c}0 \\ \frac{1}{F_{N}^{2}}\left(1-\frac{\lambda_{0}}{\lambda_{N}} \frac{u_{0}^{2}}{h_{0}}\right)\end{array}\right]$,

$\mathbf{s}_{1}=\left[-\frac{1}{F_{N}^{2}}\left[\left.\frac{\partial}{\partial h}\left(\frac{\lambda}{\lambda_{N}} \frac{u^{2}}{h}\right)\right|_{0} h_{1}+\left.\frac{\partial}{\partial u}\left(\frac{\lambda}{\lambda_{N}} \frac{u^{2}}{h}\right)\right|_{0} u_{1}\right]\right]$

with

$\left.\frac{\partial}{\partial u}\left(\frac{\lambda}{\lambda_{N}} \frac{u^{2}}{h}\right)\right|_{0}=\frac{1}{\lambda_{N} h_{0}}\left(\left.u_{0}^{2} \frac{\partial \lambda}{\partial u}\right|_{0}+2 \lambda_{0} u_{0}\right)$

$\left.\frac{\partial}{\partial h}\left(\frac{\lambda}{\lambda_{N}} \frac{u^{2}}{h}\right)\right|_{0}=\left.\frac{u_{0}^{2}}{\lambda_{N} h_{0}} \frac{\partial \lambda}{\partial h}\right|_{0}-\frac{\lambda_{0}}{\lambda_{N}} \frac{u_{0}^{2}}{h_{0}^{2}}$ 
and

$$
\begin{aligned}
& \left.\frac{\partial \lambda}{\partial u}\right|_{0}=\frac{2 a b \lambda_{0}^{3 / 2}}{d \varepsilon_{N} R e_{N} u_{0}^{2} \sqrt{\lambda_{0}}+b u_{0}\left(1-a \sqrt{\lambda_{0}}\right)} \\
& \left.\frac{\partial \lambda}{\partial h}\right|_{0}=\frac{2 a \lambda_{0}}{h_{0}} \frac{b \sqrt{\lambda_{0}}+d \varepsilon_{N} R e_{N} u_{0} \lambda_{0}}{d \varepsilon_{N} R e_{N} u_{0} \sqrt{\lambda_{0}}+b\left(1-a \sqrt{\lambda_{0}}\right)}
\end{aligned}
$$

Eqs. (22) and (23) are easily obtained from applying the implicit function theorem (Murdock, 1999) to Eq. (8).

By substituting (15)-(17) into Eq. (14), the following system is achieved:

$$
\begin{aligned}
& \kappa_{0}^{+} \frac{\partial}{\partial \tau}\left[\mathbf{u}_{0}+\mathbf{u}_{1} \tau+\mathbf{u}_{2} \tau^{2}+\ldots\right]- \\
& {\left[\mathbf{A}_{0}+\mathbf{A}_{1} \tau+\ldots\right] \frac{\partial}{\partial \tau}\left[\mathbf{u}_{0}+\mathbf{u}_{1} \tau+\mathbf{u}_{2} \tau^{2}+\ldots\right]} \\
& {\left[\mathbf{A}_{0}+\mathbf{A}_{1} \tau+\ldots\right] \frac{\partial}{\partial \xi}\left[\mathbf{u}_{0}+\mathbf{u}_{1} \tau+\mathbf{u}_{2} \tau^{2}+\ldots\right]=\mathbf{s}_{0}+\mathbf{s}_{1} \tau+\ldots}
\end{aligned}
$$

The solution of (24) at the zero-th order of $\tau$ leads to the following condition:

$u_{1}=\frac{1}{F_{N}} \frac{h_{1}}{\sqrt{h_{0}}}$

Conversely, pre-multiplying the first-order approximation of Eq. (24) with the transpose $\mathbf{l}_{0}^{+}$and accounting for the definition of left eigenvector, the following equation for $\mathbf{u}_{1}$ is deduced:

$$
\left[\kappa_{0}^{+} \frac{d \mathbf{u}_{1}}{d \xi}+\mathbf{A}_{1} \frac{d \mathbf{u}_{0}}{d \xi}-\mathbf{A}_{1} \mathbf{u}_{1}\right]^{T} \mathbf{l}_{0}^{+}=\mathbf{s}_{1}^{T} \mathbf{l}_{0}^{+}
$$

Accounting for relations (12), (18), (19), and (25) along with the continuity constraint $u_{0}=1 / h_{0}$, Eq. (26) may be rewritten in terms of the $h_{1}$ function only as follows:

$$
\frac{d h_{1}}{d \xi}-\alpha h_{1}^{2}-\gamma h_{1}=0
$$

in which:

$$
\begin{aligned}
& \alpha=\alpha(\xi)=\frac{3}{2} \frac{\sqrt{h_{0}}}{h_{0}^{3 / 2}+F_{N}} \\
& \gamma=\gamma(\xi)=\frac{h_{0}^{3 / 2}}{\left(h_{0}^{3 / 2}+F_{N}\right)}\left[\frac{1}{4 h_{0}}\left(\frac{5 F_{N}}{h_{0}^{3 / 2}}-1\right) \frac{d h_{0}}{d \xi}-r\right]
\end{aligned}
$$

where the function $r=r(\xi)$ is expressed as:

$$
\begin{aligned}
& r(\xi)=\left.\frac{1}{2 \lambda_{N} h_{0}^{3}} \frac{\partial \lambda}{\partial h}\right|_{0}-\frac{\lambda_{0}}{2 \lambda_{N}} \frac{1}{h_{0}^{4}}+ \\
& \frac{1}{F_{N} \sqrt{h_{0}}} \frac{1}{\lambda_{N} h_{0}}\left(\left.\frac{1}{2 h_{0}^{2}} \frac{\partial \lambda}{\partial u}\right|_{0}+\frac{\lambda_{0}}{h_{0}}\right)
\end{aligned}
$$

Eq. (27) represents the generalization of the findings of Dracos and Glenne (1967) to the case of variable friction coefficient, in a wide rectangular channel. It is easily recognized that Eq. (27) is a Bernoulli type equation and its analytical solution is:

$$
\frac{h_{1}(\xi)}{h_{1}(0)}=\frac{e^{\Gamma(\xi)}}{1-h_{1}(0) \int_{0}^{\xi} \alpha\left(\xi^{\prime}\right) e^{\Gamma\left(\xi^{\prime}\right)} d \xi^{\prime}} \text { with } \Gamma(\xi)=\int_{0}^{\xi} \gamma\left(\xi^{\prime}\right) d \xi^{\prime}
$$

$h_{1}(0)$ being the disturbance amplitude at the channel inlet, i.e. at $\xi=0$. It is worth noting that in Eqs. (27) and (30) both $\alpha$ and $\gamma$ functions depend on $\xi$ only through the $h_{0}$ function (Eqs. 28 and 29). Therefore, once the unperturbed gradually varied flow depth profile $h_{0}(\xi)$ is known, the perturbation spatial evolution may be evaluated through Eq. (30). However, since the equation that governs the flow depth profile:

$\frac{d h_{0}}{d \xi}=\frac{1}{\lambda_{N}} \frac{\lambda_{N} h_{0}^{3}-\lambda_{0}}{h_{0}^{3}-F_{N}^{2}}$

does not admit general closed-form solution, the $h_{0}(\xi)$ function may be known only at a discrete level and therefore the evaluation of the integrals in Eq. (30) requires a numerical quadrature.

Inspection of Eq. (30) leads to the recognition that, depending on the amplitude of $h_{1}(0)$, the $h_{1}(\xi)$ function may diverge at finite abscissa, $\xi_{b}$, and a breaking wave occurs with the formation of a shock (Witham, 1974). Downstream of the wave breaking point, the wavefront technique cannot be further applied and only the numerical solution of the full governing equations may allow the analysis of the disturbance dynamics. In a general case, the determination of the wave breaking abscissa has to be pursued numerically by solving the following equation:

$$
\int_{0}^{\xi_{b}} \alpha\left(\xi^{\prime}\right) e^{\Gamma\left(\xi^{\prime}\right)} d \xi^{\prime}=\frac{1}{h_{1}(0)}
$$

Assuming an unperturbed uniform condition, i.e. $u_{0}=h_{0}=1$, a closed-form expression of the wave breaking point may be deduced and it reads:

$\xi_{b, N}=\frac{1}{\gamma_{N}} \ln \left(1+\frac{\gamma_{N}}{\alpha_{N}} \frac{1}{h_{1}(0)}\right)$

in which $\alpha_{N}$ and $\gamma_{N}$ values follow from (28) setting $h_{0}=1$ and $\lambda_{0}=\lambda_{N}$.

Neglecting the non-linear term in Eq. (27), i.e. assuming the disturbance to be infinitesimal $\left(h_{1}(0)<<1\right)$, Eq. (30) becomes:

$\frac{h_{1}(\xi)}{h_{1}(0)}=e^{\Gamma(\xi)}$

Eq. (34), although valid for small value of the disturbance, suggests that preliminary information about the influence of the unperturbed condition on the disturbance growth may be easily inferred from the behavior of the $\gamma$ coefficient, which is uniquely defined by the unperturbed flow depth. For instance, for a given flow depth $h_{0}$, if the corresponding $\gamma$ value is smaller 
than $\gamma_{N}$, it may be expected that the spatial growth of the disturbance will be smaller than in the uniform case. Conversely, $\gamma$ values larger than $\gamma_{N}$ will lead to a larger disturbance spatial growth than in the uniform case.

Finally, assuming an unperturbed uniform condition, Eq. (34) leads the following expression of the Froude number in the marginal stability condition, i.e. $\gamma_{N}=0$ :

$$
F_{N}^{*}=\frac{2\left(a b+r^{*}\right)}{r^{*}\left(1-2 a \sqrt{\lambda_{N}}\right)-2 a^{2} \sqrt{\lambda_{N}}}
$$

where:

$$
r^{*}=d \varepsilon_{N} R e_{N} \sqrt{\lambda_{N}}+b\left(1-a \sqrt{\lambda_{N}}\right)
$$

Eq. (35) agrees with the threshold found by Di Cristo et al. (2010).

\section{FULLY NONLINEAR ANALYSIS}

The numerical solution of the dimensionless governing Equations (6)-(7) in presence of shocks has been computed starting from their corresponding conservation form:

$$
\frac{\partial \mathbf{w}}{\partial t}+\frac{\partial \mathbf{f}(\mathbf{w})}{\partial x}=\mathbf{p}(\mathbf{w})
$$

in which:

$$
\mathbf{w}=\left[\begin{array}{c}
h \\
h u
\end{array}\right] \mathbf{f}(\mathbf{w})=\left[\begin{array}{c}
h u \\
h u^{2}+\frac{1}{2} \frac{h^{2}}{F_{N}^{2}}
\end{array}\right] \mathbf{p}(\mathbf{w})=\left[\begin{array}{c}
0 \\
\frac{1}{F_{N}^{2}}\left(1-\frac{\lambda}{\lambda_{N}} u^{2}\right)
\end{array}\right]
$$

The governing Equations (37) have been herein discretized by a conventional Finite-Volume approach following the Harten-Lax-Van Leer (HLL) scheme (Harten, 1983). The values of the conserved variables at the two sides of the interface are estimated by a piecewise linear reconstruction limited by the minmod operator (Roe, 1986). The time integration is carried out according to a two-step Runge-Kutta scheme (Gottlieb and Shu, 1998). Under hypercritical flow conditions, as in the presented simulations, boundary conditions are assigned by prescribing at the channel inlet both the discharge and the flow depth.

To assess the accuracy of the complete numerical scheme, two dam-break problems and a wave evolution one have been simulated.

\section{Dam-break tests}

Two wet-bed dam-break problems have been considered to evaluate the performance of the algorithm in presence of rarefaction and shock waves. These tests, for which the Stoker (1957) solution is available, represent the benchmark of several second-order schemes considered by Zoppou and Roberts (2003). The two tests present the same water depth upstream the dam $\left(\tilde{h}_{u}=10 \mathrm{~m}\right)$ and two different downstream water depths, namely $\tilde{h}_{d}=5 \mathrm{~m}$ and $\tilde{h}_{d}=0.1 \mathrm{~m}$. In both tests, the channel length is $\tilde{L}=2000 \mathrm{~m}$ and the dam position is $\tilde{x}_{\text {dam }}=\tilde{L} / 2$. The adopted spatial discretization $(\Delta \tilde{x}=20 \mathrm{~m})$ is the same of Zoppou and Roberts (2003) and the time integration step $\Delta \tilde{t}$ has been fixed equal to $1 \mathrm{~s}$.

The performance of the present numerical method has been assessed comparing the relative error L1-norm (Zoppou and Roberts, 2003) of the computed results. Both $\tilde{h}$ and $\tilde{u}$ variables have been considered and compared with the corresponding ones of the second-order models reported in Zoppou and Roberts (2003).

For the case with $\tilde{h}_{d}=5 \mathrm{~m}$ present numerical method obtains a score of 0.65 for $\tilde{h}$ and of 0.67 for $\tilde{u}$. Both values fall within the ranges $0.29-2.27$ and $0.28-2.27$ for $\tilde{h}$ and $\tilde{u}$, respectively, of the second-order schemes considered by Zoppou and Roberts (2003). As far as the case with $\tilde{h}_{d}=0.1 \mathrm{~m}$ is concerned, the present numerical method - with a score of 0.25 for $\tilde{h}$ and of 0.24 for $\tilde{u}$ - outperforms all the second-order schemes, with the corresponding ranges $0.44-1.00$ and $0.39-1.00$ for $\tilde{h}$ and $\tilde{u}$, respectively.

\section{Wave evolution problem}

As a further benchmark, the numerical model has been applied to simulate the spatial evolution of a time-periodic perturbation of the flow discharge at the channel inlet, in the neighborhood of uniform flow. In what follows $\alpha_{p}$ denotes the amplitude of the perturbation whose pulsation is $\omega=2 \pi / T, T$ being the perturbation period. For very small $\alpha_{p}$ values the problem may be linearized. Assuming a constant friction factor, the analytical solution is known (Supino, 1960) and it was used by Zanuttigh and Lamberti (2002) to assess the performance of their numerical method. Denoting with $q_{p}(x, t)<<1$ and $h_{p}(x, t)<<1$ the perturbation of discharge and flow depth respect to the corresponding uniform values, it is easy to verify that the extension of the analytical solution of Supino (1960), for the resistance model expressed by Eq. (8), reads:

$$
\begin{aligned}
q_{p}(x, t) & =\frac{\alpha_{p}}{2}\left\{e^{\psi^{+} x} \sin \left[\omega\left(t-\frac{x}{\kappa_{0}^{+}}\right)\right]+e^{\psi^{-} x} \sin \left[\omega\left(t-\frac{x}{\kappa_{0}^{-}}\right)\right]\right\} \\
h_{p}(x, t) & =\frac{\alpha_{p}}{2}\left\{\psi^{+} e^{\psi^{+} x}\left(\frac{1}{\kappa_{0}^{+}} \sin \left[\omega\left(t-\frac{x}{\kappa_{0}^{+}}\right)\right]+\frac{1}{\omega} \cos \left[\omega\left(t-\frac{x}{\kappa_{0}^{+}}\right)\right]\right)+\right. \\
& \left.+\psi^{-} e^{\psi^{-} x}\left(\frac{1}{\kappa_{0}^{-}} \sin \left[\omega\left(t-\frac{x}{\kappa_{0}^{-}}\right)\right]+\frac{1}{\omega} \cos \left[\omega\left(t-\frac{x}{\kappa_{0}^{-}}\right)\right]\right)\right\}
\end{aligned}
$$


in which:

$$
\psi^{ \pm}=\frac{\sqrt{j+\sqrt{j^{2}+w}} \mp 2\left(3 \lambda_{N}-\left.\frac{\partial \lambda}{\partial h}\right|_{N}\right)}{4 \lambda_{N}\left(F_{N}^{2}-1\right)}
$$

and:

$$
\begin{aligned}
& j=\left[18-8\left(F_{N}^{2} \omega\right)^{2}\right] \lambda_{N}^{2}+\left.2\left(\left.\frac{\partial \lambda}{\partial h}\right|_{N}-6\right) \frac{\partial \lambda}{\partial h}\right|_{N} \lambda_{N} \\
& w=\left(8 \omega \lambda_{N}\right)^{2}\left[-\left(2+F_{N}^{2}\right) \lambda_{N}+\left.\left(2 F_{N}^{2}-1\right) \frac{\partial \lambda}{\partial h}\right|_{N}+\left.\frac{\partial \lambda}{\partial u}\right|_{N}\right]
\end{aligned}
$$

Analytical and numerical solutions have been compared considering the dimensionless channel length $L=25$. The $R e_{N}$ and $\varepsilon_{N}$ values have been fixed equal to $5 \cdot 10^{4}$ and $1 \cdot 10^{-2}$, respectively. The following Colebrook-White coefficients have been assumed: $a=-2.03 / \ln (10), b=0.85, d=1 / 10.95$ (Zanuttigh and Lamberti, 2002). The corresponding marginal stable Froude number (Eq. 35) is $F_{N}^{*}=1.533$. The perturbation amplitude has been assigned equal to $1 \cdot 10^{-3}$ and the period is $T=8$. Numerical simulations have been carried out with $\Delta x=L / 800$ and $\Delta t=1 / 64$. Three different values of the Froude number, i.e. $F_{N}=0.75 F_{N}^{*}, F_{N}=F_{N}^{*}$ and $F_{N}=1.5 F_{N}^{*}$, have been considered in order to test the algorithm in stable, marginally stable and unstable conditions. The relative error L1-norm on flow depth and discharge over a period has been found to vary in all tests in the range $\left(10^{-6}, 10^{-5}\right)$. Therefore, an excellent agreement between numerical and analytical solutions has been observed, independently of the Froude number. As an example, the exact and computed solutions are compared in Figure 1 for the $F_{N}=1.5 F_{N}^{*}$ case in terms of $\hat{h}_{p}(x, t)=h_{p}(x, t) / h_{p}(0,0)$.

\section{RESULTS AND DISCUSSION}

The present section discusses the influence of the inlet boundary condition on the roll-waves development in gradually varied hypercritical profiles with reference to two of the five tests reported in Zanuttigh and Lamberti (2002), i.e. Tests 2 and 5 , here referred as Tests A and B, respectively. Assuming uniform unperturbed flow condition, Zanuttigh and Lamberti (2002) showed that the hyperbolic SVEs are able to reproduce all the essential features of the roll-waves observed in the Brock (1967) experiments.

The channel is rectangular, $80 \mathrm{~m}$ long and $0.1175 \mathrm{~m}$ wide. For both tests, the ratio of the hydraulic radius to the flow depth in uniform flow conditions is about 0.9 and therefore the hypothesis of wide rectangular channel may be safely assumed. The hydraulic parameters for both tests are reported in Table 1, while Table 2 contains the values of the relevant dimensionless parameters computed assuming the same values of the Colebrook-White coefficients considered in the previous section. In the same table the dimensionless critical flow depth $h_{c}=F_{N}^{2 / 3}$ and the dimensionless channel lengths $(L)$ values are also reported, along with the values of the Froude number $\left(F_{N}^{*}\right)$ in marginal linearly stable flow condition (Eq. 35). Tables 1 and 2 indicate that both tests refer to steep slope channels, i.e. $h_{\mathrm{c}}>h_{N}$, and to linearly unstable uniform conditions, i.e. $F_{N}>F_{N}^{*}$.
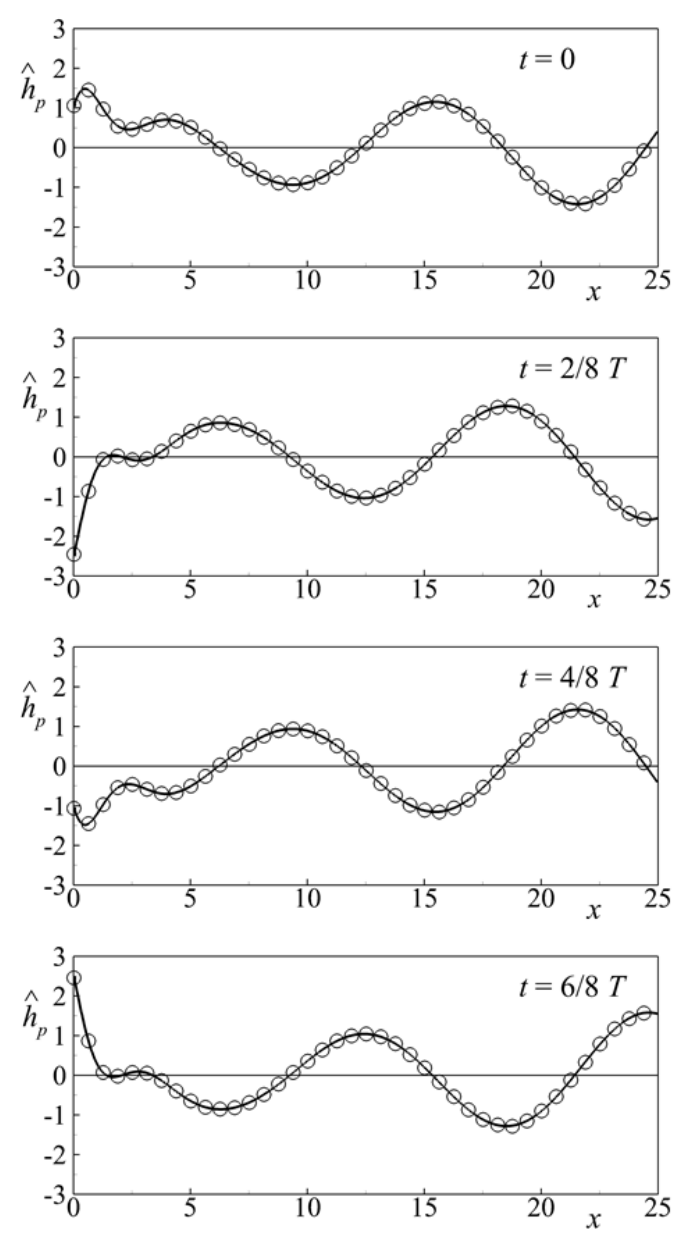

Fig. 1. Comparison between analytical (solid line) and numerical (symbols) solution. $F_{N}=1.5 F_{N}^{*}$.

Table 1. Hydraulic parameters of the considered tests.

\begin{tabular}{c|c|c|c|c}
\hline Test & $\begin{array}{c}\sin (\theta) \\
{[-]}\end{array}$ & $\begin{array}{c}\tilde{h}_{N} \\
{[\mathrm{~m}]}\end{array}$ & $\begin{array}{c}\tilde{u}_{N} / \sqrt{g \tilde{h}_{N}} \\
{[-]}\end{array}$ & $\begin{array}{c}\lambda_{N} \\
{[-]}\end{array}$ \\
\hline $\mathrm{A}$ & 0.05011 & $7.97 \cdot 10^{-3}$ & 3.71 & 0.0257 \\
\hline $\mathrm{B}$ & 0.11920 & $5.33 \cdot 10^{-3}$ & 5.60 & 0.0279 \\
\hline
\end{tabular}

Table 2. Dimensionless parameters.

\begin{tabular}{c|c|c|c|c|c|c}
\hline Test & $F_{N}$ & $R e_{N}$ & $\varepsilon_{N}$ & $h_{c}$ & $L$ & $F_{N}^{*}$ \\
\hline $\mathrm{A}$ & 3.71 & $3.2810^{4}$ & $7.5010^{-3}$ & 2.40 & 510 & 1.53 \\
\hline $\mathrm{B}$ & 5.62 & $2.7110^{4}$ & $1.0210^{-2}$ & 3.16 & 1500 & 1.52 \\
\hline
\end{tabular}

For each test, keeping fixed the flow rate and imposing at the channel inlet a flow depth different from the uniform one, the effects of a gradually varied profile on the roll-wave evolution has been synthetically investigated. In what follows both accelerated (S2, $1<h_{0}<h_{\mathrm{c}}$ ) and decelerated (S3, $\left.0<h_{0}<1\right)$ hypercritical profiles are considered.

For each test five profiles, two accelerated and two decelerated along with the uniform one, have been considered. As far as the S2 profiles are concerned, the first boundary condition has been fixed equal to a value slightly smaller than the critical flow depth (profile S2 ; Test A: $h_{0, U}=2.1$, Test B: $h_{0, U}=2.9$ ); the second one to an intermediate value between the critical and the uniform flow depths (profile S2 $2_{\text {II }}$; Test A: $h_{0, U}=1.6$, Test B: 
$h_{0, U}=2.0$ ). The flow depths at the channel inlet for the $\mathrm{S} 3 \mathrm{de}-$ celerated profiles have been assumed equal to $h_{0, U}=0.50$ (profile $\mathrm{S} 3_{\mathrm{I}}$ ) and $h_{0, U}=0.25$ (profile $\mathrm{S} 3_{\mathrm{II}}$ ), for both tests. All profiles have been determined numerically integrating Eq. (31) through a standard second-order Runge-Kutta method with a resolution equal to $\Delta \xi=\Delta x=1 \cdot 10^{-1}$. Figure 2 reports the computed results, in which, only for the sake of clarity, the channel length has not been entirely represented. For both tests, the uniform conditions $(\mathrm{N})$ are practically recovered relatively close to the channel inlet. Indeed, while for Test A the uniform condition is reached for $\xi$ values larger than 15-20 dimensionless channel units, in Test $\mathrm{B}$ a larger distance from the channel inlet is required $(\xi \approx 35-40)$.

In the two next subsections the influence of the unperturbed profiles on the roll-wave development is explored either through the near wavefront expansion analysis or through the numerical simulation of SVEs.
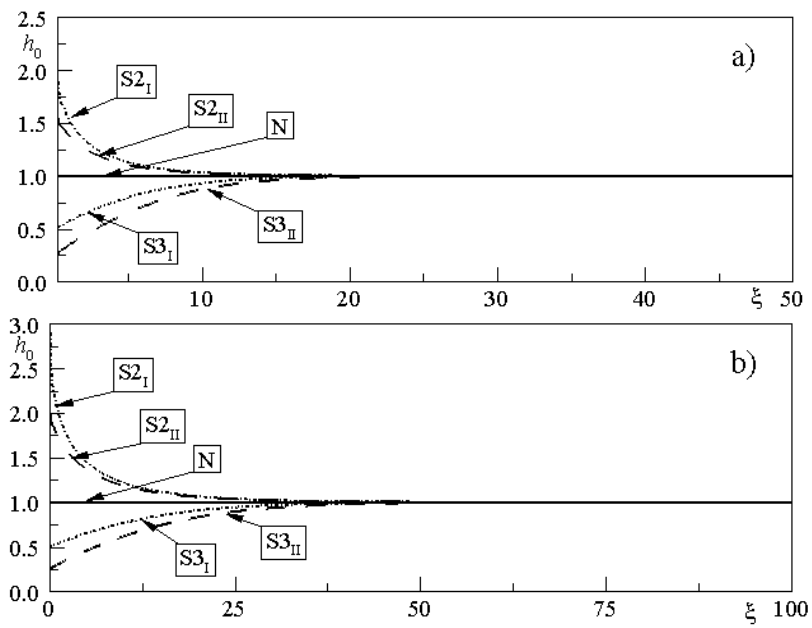

Fig. 2. Gradually varied profiles: a) Test A; b) Test B.

\section{Spatial evolution of a pointwise disturbance}

In order to investigate the influence on the disturbance development of unperturbed gradually varied profile, the results of the linear analysis are preliminarily discussed. To this aim, Figure 3 depicts, for hypercritical conditions, the variation of $\gamma$ coefficient with the flow depth $h_{0}$.

As explained in section 3, the behavior of the $\gamma$ coefficient furnishes some useful information on the disturbance spatial growth. Despite the inherent limitation of the linearized theory, Figure 3 suggests the following conclusions. As expected, for both tests in uniform flow condition, i.e. $h_{0}=1$, a positive value of $\gamma_{N}$ has been found, i.e. $\gamma_{N}=7.9610^{-2}$ (Test A) and $\gamma_{N}=$ $7.1010^{-2}$ (Test B). The flow depth value $\left(\hat{h}_{0}\right)$ for which $\gamma$ vanishes is larger than one, i.e. $\hat{h}_{0} \approx 1.17$ (Test $\mathrm{A}$ ) and $\hat{h}_{0} \approx 1.28$ (Test B), and all positive values of the $\gamma$ coefficient are found for $h_{0}<\hat{h}_{0}$, while negative ones are encountered for $\hat{h}_{0}<h_{0}<h_{c}$. It is worth of note that for flow depth very close to the critical value or to the vanishing one, for which the expansion near the wavefront predicts that $\gamma$ diverges, present results have to be taken with caution, owing to the basic assumptions behind the SVEs.

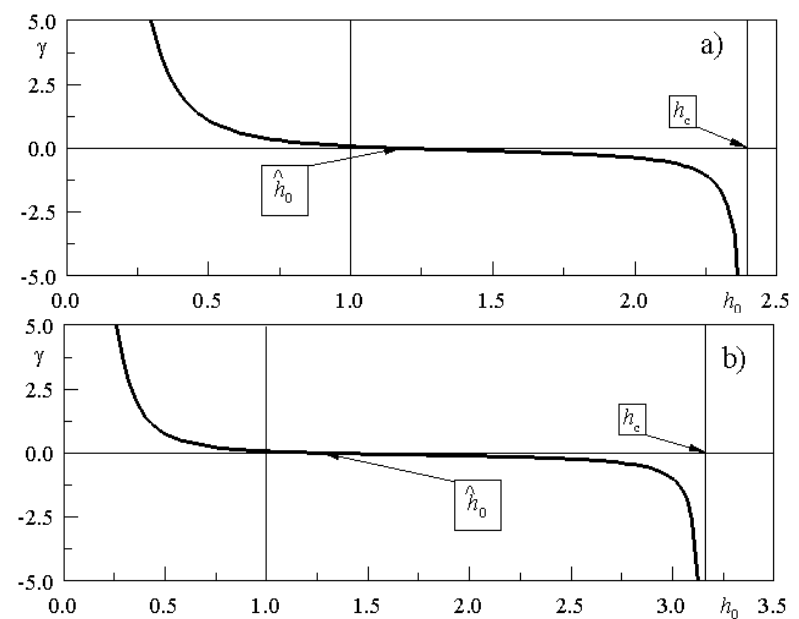

Fig. 3. Flow depth dependence of the $\gamma$ coefficient. a) Test A; b) Test B.

Figure 3 suggests also that, at least for very small disturbance, flow depths smaller than the uniform value may induce a disturbance growth larger than the one related to the uniform condition. Conversely, for flow depth $1<h_{0}<\hat{h}_{0}$ the disturbance growth is smaller than in uniform condition. Finally, for $\hat{h}_{0}<h_{0}<h_{c}$ an amplitude reduction is expected. This behavior is related to the sign of the first term in the $\gamma$ definition (i.e. Eq. (28)). In fact, since for hypercritical flow depth the term $F_{N} / h_{0}^{3 / 2}=\left(h_{c} / h_{0}\right)^{3 / 2}$ is always larger than one, the sign of first term in the $\gamma$ definition only depends on the $d h_{0} / d \xi$ sign, which is positive for $0<h_{0}<1$ and negative for $1<h_{0}<h_{\mathrm{c}}$.

The results of the linearized model suggest that the unperturbed profile could exert a non-negligible effect on the perturbation spatial evolution also in the non-linear case.

In order to confirm such a dependence and with reference to the gradually profiles represented in Figure 2 the spatial evolution of the perturbation has been evaluated numerically integrating Eq. (30) with the trapezoidal rule. The perturbation magnitude has been initially set equal to $h_{1}(0)=10^{-4}$. The computed spatial evolution of the perturbation is reported in Figure 4 for both tests and all considered profiles, in terms of $\beta(\xi)=h_{1}(\xi) / h_{1}(0)$.

For uniform conditions (N) and for $\xi$ smaller than about 80 , Figures $4 \mathrm{a}$ and $4 \mathrm{~b}$ show an exponential disturbance growth, with a rate equal to $\gamma_{N}$. Downstream 80 channel units, owing to the equation non-linearity, a more-than-exponential growth of the disturbance is observed, leading finally to the wave breaking. The wave breaking abscissas $\xi_{b, N}$ agree with the analytical values given by Eq. (33), i.e. $\xi_{b, N}=98.24$ (Test A) and $\xi_{b, N}=113.40$ (Test B).

As far as the Test $\mathrm{A}$ is concerned, Figure 4a indicates that both the decelerated S3 profiles enhance the growth rate of the spatial disturbance close to the channel inlet, with respect to the uniform condition. In the $\mathrm{S} 3_{\mathrm{I}}$ case, the growth becomes exponential with a rate equal to $\gamma_{N}$ for abscissa larger than about 20 channel units. Such a feature may be explained accounting for that, for $\xi$ larger than about 20 , the flow depth has essentially reached the uniform value (Figure 2) and the non-linear term in Eq. (27) has only a marginal role. 


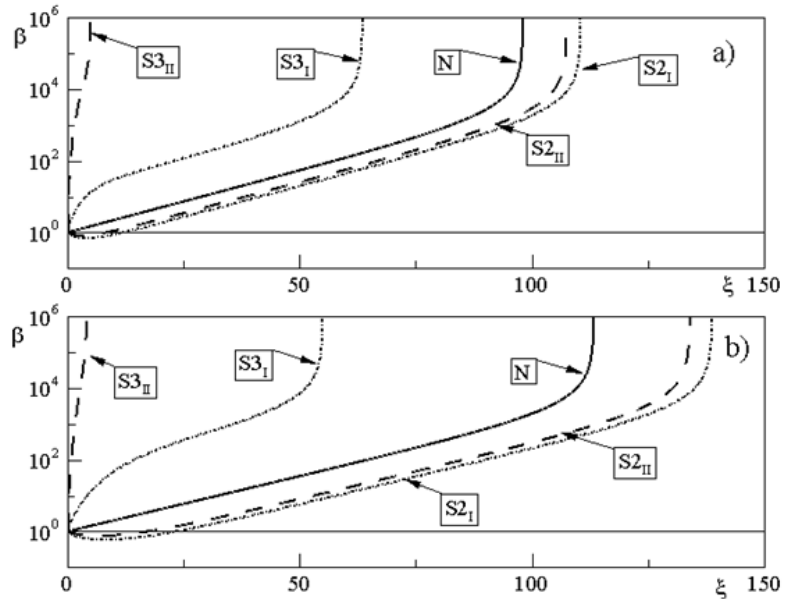

Fig. 4. Disturbance spatial evolution. a) Test A; b) Test B.

The onset of non-linearity occurs for $\xi$ larger than about 50 units, inducing a wave breaking at $\xi_{b} \approx 64$. In the $\mathrm{S} 3_{\text {II }}$ profile, the effect of the inlet condition on the spatial growth is so strong that the wave breaking occurs very close to the channel inlet, i.e. for $\xi_{b} \approx 5$, and the exponential growth cannot take place. Conversely, for both the accelerated S2 profiles, close to the channel inlet, the disturbance amplitude reduces in the very first channel units, then it increases recovering its initial value at the abscissa $\xi_{0}$ (with $\xi_{0} \cong 8$ in the $\mathrm{S} 2_{\text {II }}$ profile and $\xi_{0} \cong 11$ in the $\mathrm{S} 2_{\mathrm{I}}$ one). Downstream the abscissa $\xi_{0}$, a monotone disturbance growth is predicted, which is initially exponential with a growth rate $\gamma_{N}$, then it further increases up to the wave breaking. The wave breaking occurs at larger distance from the channel inlet in the $\mathrm{S} 2_{\mathrm{I}}$ case than in the $\mathrm{S} 2_{\mathrm{II}}$ one $\left(\mathrm{S} 2_{\mathrm{I}}: \xi_{b} \approx 111\right.$; $\mathrm{S} 2_{\text {III }}: \xi_{b} \approx 108$ ).

From the qualitative point of view, the Test B (Figure 4b) exhibits the same behavior discussed for Test A. From a quantitative point of view, Figure $4 \mathrm{~b}$ indicates that in the case of the decelerated profiles the wave breaking occurs closer to the channel inlet than in the Test $\mathrm{A}\left(\mathrm{S}_{\mathrm{I}}: \xi_{b} \approx 55 ; \mathrm{S} 3_{\mathrm{II}}: \xi_{b} \approx 4\right)$, while the breaking abscissas increase in the accelerated cases $\left(\mathrm{S} 2_{\mathrm{I}}: \xi_{b} \approx 134 ; \mathrm{S} 2_{\mathrm{II}}: \xi_{b} \approx 140\right)$.

In order to investigate the effect of the perturbation magnitude, only the $\mathrm{S} 3_{1}$ and $\mathrm{S} 2_{2}$ cases of the Test $\mathrm{B}$, along with the uniform one, have been considered. For each profile, the disturbance evolution has been computed considering three different values of $h_{1}(0)$, namely $10^{-6}, 10^{-2}$ and $10^{-1}$. The computed results are shown in Figure 5, which for sake of comparison, includes also the $h_{1}(0)=10^{-4}$ case.

Figure 5 suggests that a reduction of the disturbance magnitude enlarges the portion of the channel in which the linear theory is applicable, shifting downstream from the channel inlet the wave breaking point. Conversely, an amplitude increase produces an upstream shift of the wave breaking point.

For the decelerated profile, Figure 5c shows that if high values of the disturbance at the inlet are prescribed, the initial exponential growth phase may not take place.

All the above results taken collectively indicate that the unperturbed profile has a strong influence on the spatial evolution of a single perturbation. In particular, a positive free surface slope in the unperturbed profile induces a disturbance spatial growth larger than in the uniform condition, translating upstream from the channel inlet the wave breaking. A negative free surface profile induces a downstream translation of wave

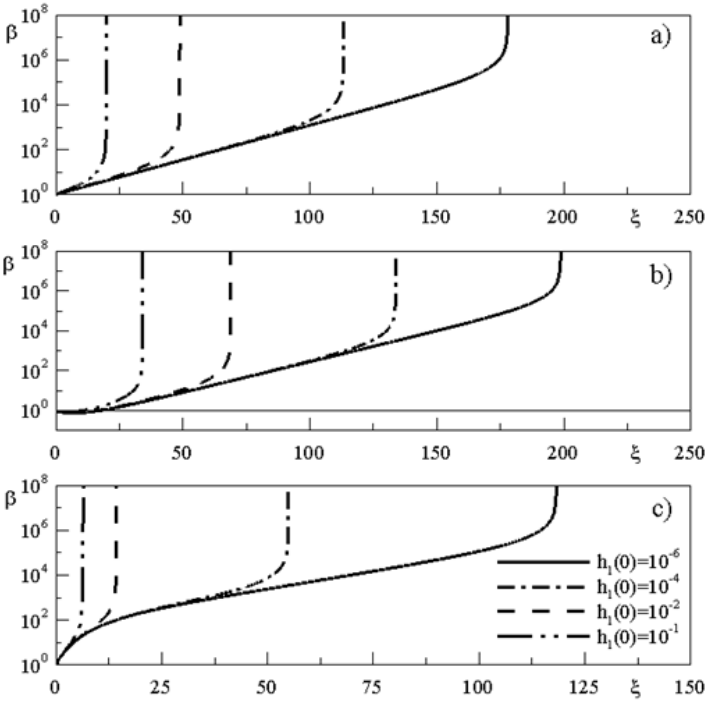

Fig. 5. Effect of the perturbation magnitude on the disturbance spatial evolution (Test B). a) Uniform profile; b) Accelerated S2 $2_{\text {II }}$ profile; c) Decelerated $\mathrm{S}_{\mathrm{I}}$ profile.

breaking. Such a spatial shift of the perturbation evolution is expected to have a non-negligible influence also on the spatial evolution of roll-wave trains.

\section{Spatial evolution of roll-wave trains}

In the present subsection the results of the numerical simulations of the roll-wave train evolution, with reference to both Tests A and B, are presented and discussed. All hypercritical unperturbed profiles of the previous section have been considered.

With the aim of reproducing the natural roll-waves experimented by Brock (1967), Zanuttigh and Lamberti (2002) numerically solved the hyperbolic SVEs, with unperturbed uniform conditions, adding random small perturbations at the channel inlet. Comparing their numerical results with the experimental ones of Brock (1967), Zanuttigh and Lamberti (2002) have shown that a fair agreement among Brock's experiments and computations was achieved provided that the mesh spacing was in the range $\Delta x=6 \div 10 h_{N}$ and a suitable value of the r.m.s. intensity perturbation at the channel inlet was applied. Indeed their numerical results were able to reproduce both instabilities of the uniform regime in the initial developing phase and the wave overtaking in the final phase, both observed in the Brock (1967) experiments.

In the present simulations, the computational grids for both tests have been therefore defined accounting for the Zanuttigh and Lamberti (2002) findings. Indeed since the suggested dimensionless mesh spacing range for the Test $\mathrm{A}$ is $0.3-0.5$, it is assumed $\Delta x=0.34$. For the Test $\mathrm{B}$ the mesh spacing has been fixed equal to $\Delta x=1.15$, within the suggested range $0.72-1.2$. The r.m.s. intensity at the inlet has been defined in order to reproduce, in uniform unperturbed conditions, the dimensionless statistical characteristics considered by Zanuttigh and Lamberti (2002): the mean water depth at crest $h_{\max }^{\prime}$, the mean water depth at trough $h_{\min }^{\prime}$ and the mean wave period $T^{\prime}$. Statistics were collected on the second half of a dimensionless time window of 8192 (Test A) and 32768 (Test B) dimensionless time units, discarding the first half to cancel the effects of the initial condition. This time window guaranteed the evaluation 
of statistic properties on more than 500 waves, detected accordingly to the zero down-crossing method. The dimensionless value r.m.s. intensity of the inlet disturbance has been fixed equal to $5 \cdot 10^{-4}$ (Test A) and to $5 \cdot 10^{-3}$ (Test B). For uniform unperturbed conditions, Figures 6 and 7 report the computed $h_{\max }^{\prime},-h_{\min }^{\prime}$ and $T^{\prime}$ spatial distributions, for the Tests A and B, respectively, along with the Zanuttigh and Lamberti (2002) results. The agreement with the reference data is satisfactory, for all the statistical properties and for both tests.
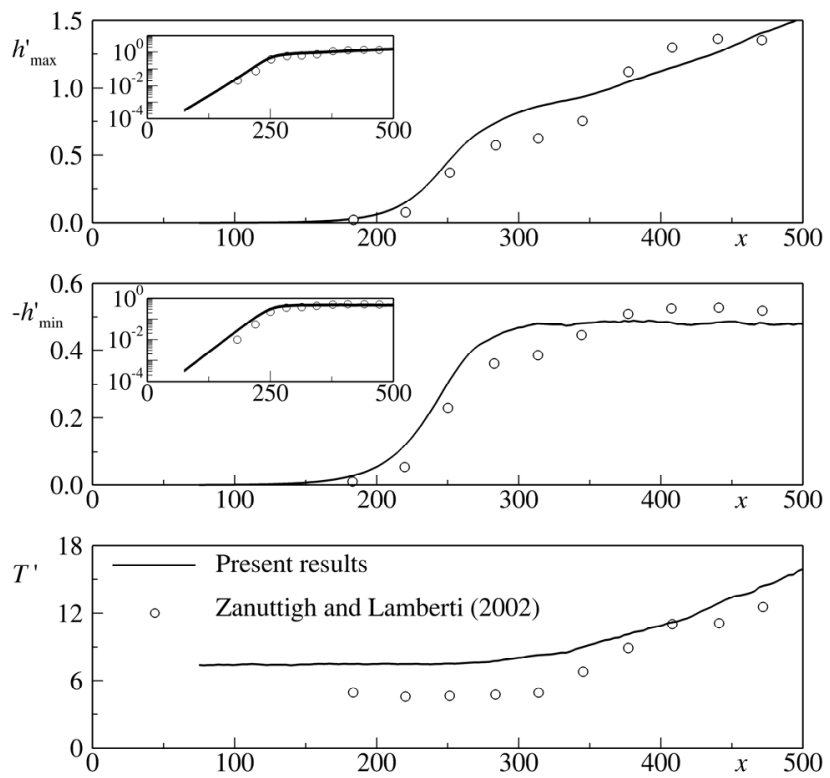

Fig. 6. Computed and literature reference values of the statistical parameters in uniform condition for Test A (inset in semilogarithmic coordinates).
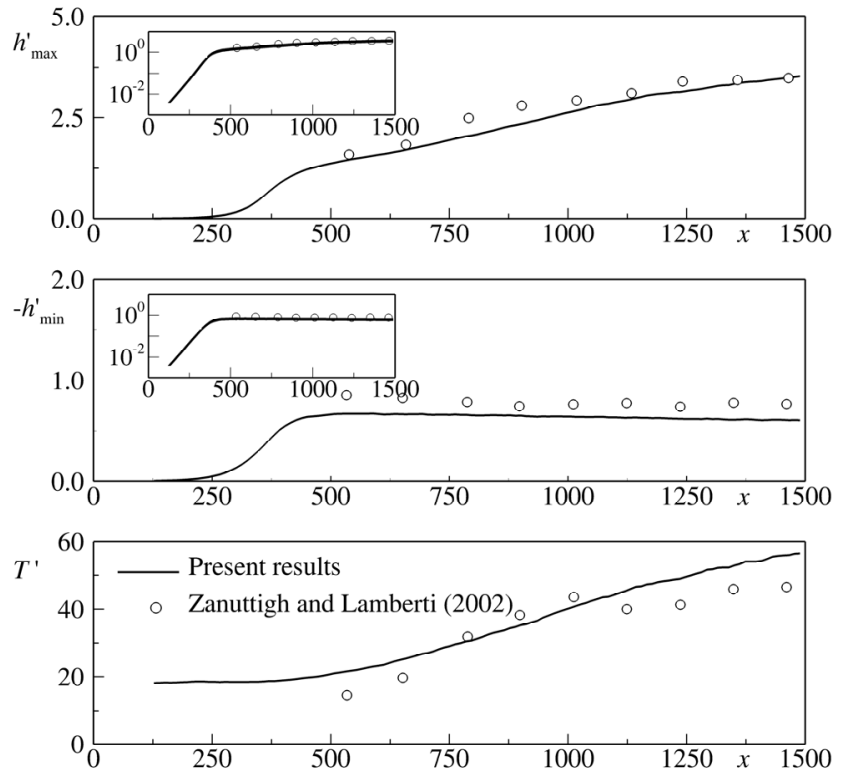

Fig. 7. Computed and literature reference values of the statistical parameters in uniform condition for Test B (inset in semilogarithmic coordinates).

The presence of the three different phases, peculiar of the natural roll-waves development, is enlightened by Figures 6 and 7. In the first phase, i.e. initial or instability phase, the waves, small and symmetric, are characterized by an exponential amplitude growth and an almost constant mean wave period $T^{\prime}$. In the second phase, known as the transition one, the mean water depth at trough $h_{\text {min }}^{\prime}$ rapidly reaches its final constant value, $h_{\max }^{\prime}$ linearly increases and the wave period $T^{\prime}$ begins to increase. In the third or final phase $h_{\min }^{\prime}$ is nearly constant, $T^{\prime}$ quasi-linearly grows and $h_{\max }^{\prime}$ increases but with a growth rate decreasing along the channel.

Keeping fixed the mesh space resolution and the r.m.s. intensity, simulations with accelerated and decelerated unperturbed profiles have been performed. Figures 8 and 9 show the spatial evolution of $h_{\max }^{\prime},-h_{\min }^{\prime}$ and $T^{\prime}$ for Test A with the accelerated $\left(\mathrm{S} 2_{\mathrm{I}}\right.$ and $\left.\mathrm{S} 2_{\mathrm{II}}\right)$ and decelerated $\left(\mathrm{S} 3_{\mathrm{I}}\right.$ and $\left.\mathrm{S} 3_{\mathrm{II}}\right)$ profiles, respectively. For the sake of comparison in Figures 8 and 9 the results pertaining to the uniform unperturbed condition (N) are also reported.

As suggested by the expansion near a wavefront technique, Figure 8a indicates that the accelerated profiles induce a smaller roll-waves spatial growth than the uniform one. Such a feature is particularly evident for the $\mathrm{S} 2_{\mathrm{I}}$ profile. Both initial and transition phases appear to be shifted of a length $L_{\mathrm{s}}$ in the downstream direction, with $L_{\mathrm{s}} \sim 100$ for the $\mathrm{S} 2_{\mathrm{I}}$ profile and $L_{\mathrm{s}} \sim$ 60 for the $\mathrm{S} 2_{\mathrm{II}}$ one. It is worth noting that both $L_{\mathrm{s}}$ values largely exceed the distance at which the two profiles recover the uniform one (see Figure 2). In the final phase the linear growth of the $h_{\max }^{\prime}$ distribution occur with the same rate of the uniform case. As matter of fact, at the channel end the maximum value of $h_{\max }^{\prime}$ for the accelerated profiles is smaller than the one with uniform condition. The $-h_{\min }^{\prime}$ spatial distributions show an analogous downstream-shift reaching a maximum value at the end of the transition phase, which is independent on the inlet condition. In the initial phase, the mean wave period $T^{\prime}$ distribution for both $\mathrm{S} 2_{\mathrm{I}}$ and $\mathrm{S} 2_{\mathrm{I}}$ profiles overwhelms the corresponding one in uniform condition. Moreover, differently from the uniform case, in such a phase $T^{\prime}$ is non-constant along the channel but it reduces. Since the $T^{\prime}$ growth rates in the transition and final phases in uniform condition is larger than the one of the accelerated profiles, at the channel end the mean wave period in uniform condition is the largest one.

In agreement with what suggested by the near wavefront expansion, in the initial phase the decelerated $\mathrm{S} 3_{\text {I }}$ and $\mathrm{S} 3_{\text {II }}$ profiles (Figure 9) induce a larger growth of $h_{\max }^{\prime}$ than the uniform one. Compared with the uniform case, an upstream-shift of the $h_{\max }^{\prime}$ distributions is detected. The largest shift pertains to the $\mathrm{S} 3_{\text {II }}$ case. Therefore, the effect of the decelerated profiles is opposite to the one of the accelerated profile, although less pronounced. The final $h_{\max }^{\prime}$ value, attained at the end of the transition phase, essentially coincides with the one pertaining to the uniform condition, for both S3 profiles. Consequently, the $h_{\max }^{\prime}$ spatial distribution in the final phase is profileindependent. The $-h_{\min }^{\prime}$ spatial distributions show a similar upstream-shift, reaching at the end of the transition phase a constant value, which is profile-independent, similarly to the accelerated cases. The constancy of the mean wave period $T^{\prime}$ in initial phase is almost preserved, although with a value larger than with uniform inlet conditions. However, at the end of the transition phase and during the final phase the spatial $T^{\prime}$ distribution of both $\mathrm{S} 3_{\mathrm{I}}$ and $\mathrm{S} 3_{\mathrm{II}}$ closely follows the uniform one. 

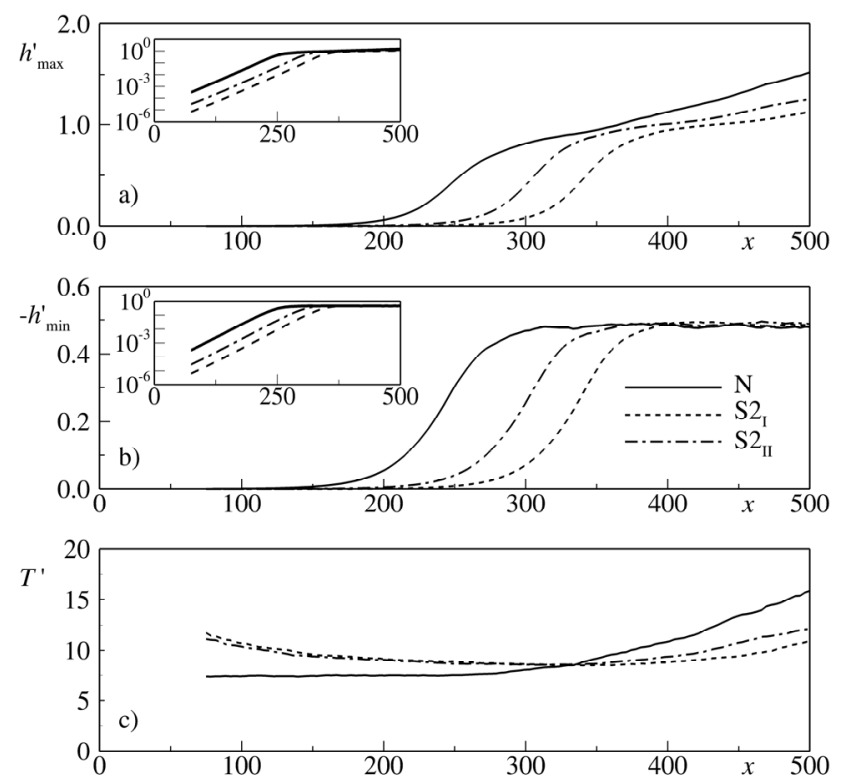

Fig. 8. Simulated spatial evolution of the statistical parameters for accelerated profiles (Test A). a) Mean water depth at crest (inset in semi-logarithmic coordinates); b) Mean water depth at trough (zoom in semi-logarithmic coordinates); c) Mean wave period.
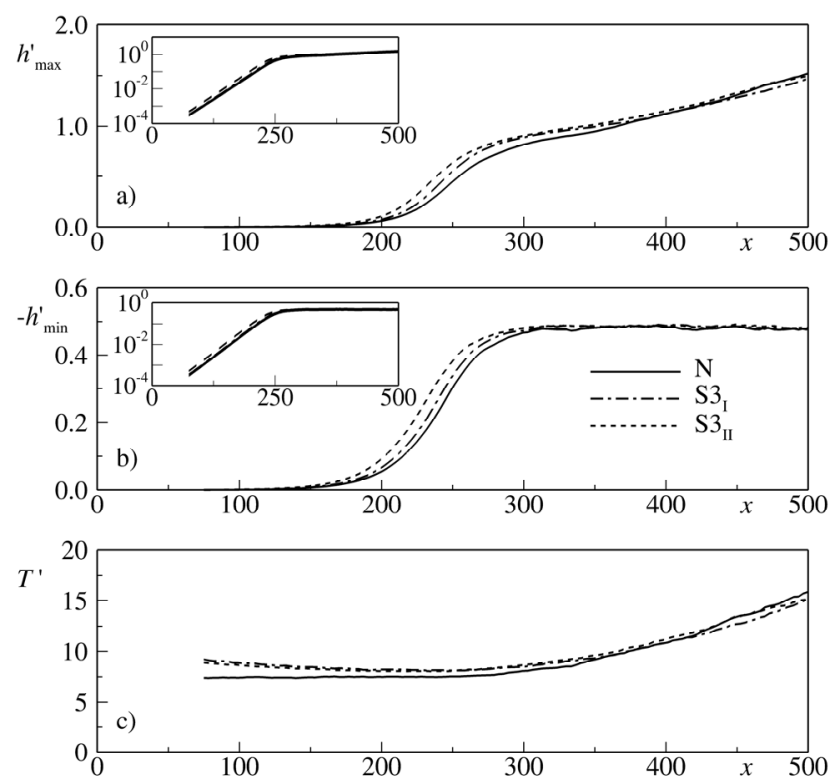

Fig. 9. Simulated spatial evolution of the statistical parameters for decelerated profiles (Test A). a) Mean water depth at crest (inset in semi-logarithmic coordinates); b) Mean water depth at trough (zoom in semi-logarithmic coordinates); c) Mean wave period.

Figures 10 and 11, which are the counterparts of Figures 8 and 9 for the Test B, endorse the information provided by Test A from the qualitative point of view. The main difference concerns the accelerated cases (Figure 10) for which the shift respect to the uniform condition is highly magnified $\left(L_{\mathrm{s}} \sim 250\right.$ for the $\mathrm{S} 2_{\text {I }}$ profile and $L_{\mathrm{s}} \sim 100$ for the $\mathrm{S} 2_{\mathrm{II}}$ one).

From the presented results, it may be therefore concluded that both the accelerated and decelerated profiles have a strong impact on the roll-wave train development.
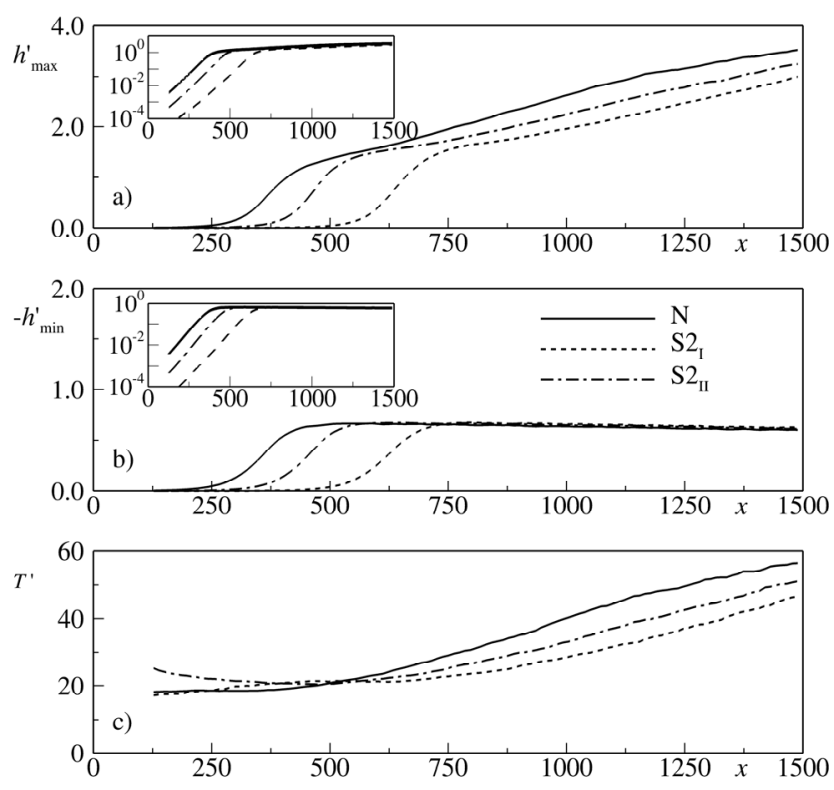

Fig. 10. Simulated spatial evolution of the statistical parameters for accelerated profiles (Test B). a) Mean water depth at crest (inset in semi-logarithmic coordinates); b) Mean water depth at trough (zoom in semi-logarithmic coordinates); c) Mean wave period.
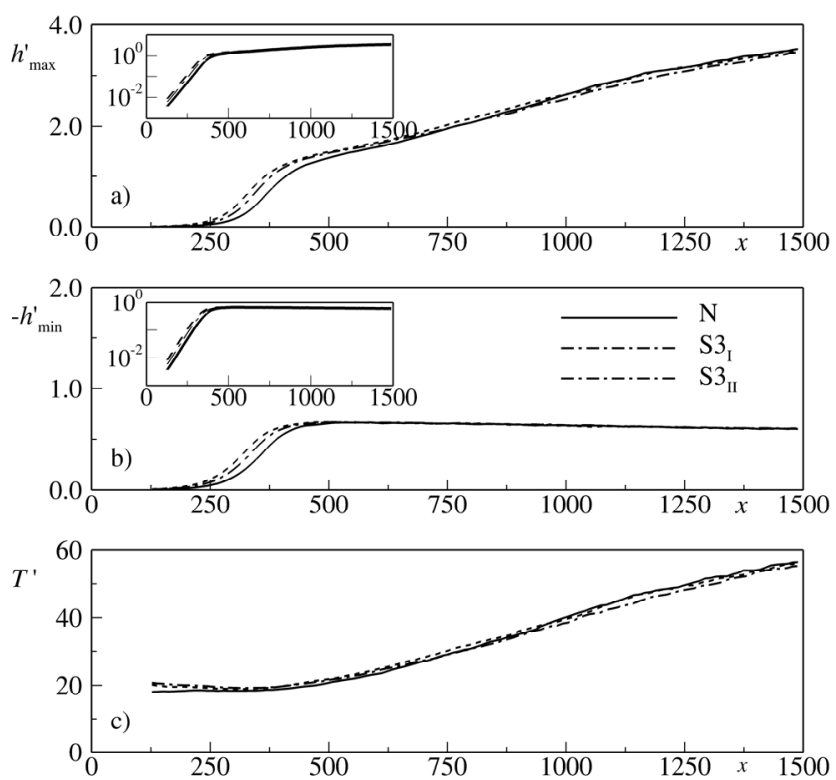

Fig. 11. Simulated spatial evolution of the statistical parameters for decelerated profiles (Test B). a) Mean water depth at crest (inset in semi-logarithmic coordinates); b) Mean water depth at trough (zoom in semi-logarithmic coordinates); c) Mean wave period.

As far as the initial phase is concerned, downward shift is caused by an accelerated profile in both $h_{\max }^{\prime}$ and $h_{\min }^{\prime}$ spatial distributions. Conversely, a decelerated profile causes an upward shift. The roll-waves dynamics in the transition and final phases leads to an asymptotic value $h_{\min }^{\prime}$ which is profileindependent. Such a conclusion holds even for asymptotic value of the mean water depth at crest but only in presence of decelerated profiles. In contrast, accelerated profiles cause $h_{\max }^{\prime}$ values that are always smaller than the corresponding in 
uniform conditions. The influence of inlet condition on the mean wave period is appreciable only with reference to decelerated profiles which induce, in the final phase, a reduction of $T^{\prime}$ with respect to the uniform condition.

\section{CONCLUSIONS}

The paper theoretically and numerically investigated the effect of gradually varying profiles on the roll-waves evolution in turbulent clear-water flows. The theoretical analysis, performed through the near wavefront expansion technique, accounts for the variability of the friction coefficient. The study of the spatial evolution of a single perturbation has shown that the growth of the perturbation is amplified (dampened) when it propagates over a decelerated (accelerated) hypercritical profile. The numerical simulation of natural roll-wave trains, carried out through a second-order Finite Volume Method has confirmed this effect, for both the initial and the transition phases. The differences with the uniform profile still persist in the final phase for the accelerated profiles, while they strongly fade out for the decelerated ones. The effect of the inlet profile should be therefore carefully considered in the estimation of the spatial growth rate used in the criteria for predicting roll-wave appearance.

\section{REFERENCES}

Balmforth, N.J., Mandre, S., 2004. Dynamics of roll waves. J. Fluid Mech., 514, 1-33.

Berlamont, J.F., 1976. Roll-waves in inclined rectangular open channels, A2, BHRA Fluid Eng., Newcastle, U.K., 13-26.

Berlamont, J.F., Vanderstappen, N., 1981. Unstable turbulent flow in open channels. J. Hydraul. Div., 1074, 427-449.

Brock, R., 1967. Development of roll waves in open channels. W.M. Keck Lab. of Hydraul. and Water Resources, California Inst. of Tech., Report KH-R-16, 226.

Brock, R., 1969. Development of roll-wave trains in open channel. J. Hydraul. Div. ASCE, 95, HY4, 1401-1427.

Brock, R., 1970. Periodic permanent roll waves. J. Hydraul. Div., 9612, 2565-2580.

Cao, Z., Hu, P., Hu, K., Pender, G., Liug Q., 2015. Modelling roll waves with shallow water equations and turbulent closure. J. Hydraul. Res., 53, 2, 161-177.

Chanson, H., 2004. The Hydraulics of Open Channel Flow: an Introduction. Elsevier, Amsterdam.

Di Cristo, C., Vacca, A., 2005. On the convective nature of roll waves instability. J. Appl. Math., 2005, 259-271.

Di Cristo, C., Iervolino, M., Vacca, A., Zanuttigh, B., 2008. Minimum channel length for roll-waves generation. J. Hydraul. Res., 46, 1, 73-79.

Di Cristo, C., Iervolino, M., Vacca, A., Zanuttigh, B., 2010. Influence of relative roughness and Reynolds number on the roll waves spatial evolution. J. Hydraul. Eng., 136, 1, 24-33.

Di Cristo, C., Iervolino, M., Vacca, A., 2012a. Green's function of the linearized Saint-Venant equations in laminar and turbulent flows. Acta Geophysica, 60, 1, 173-190.

Di Cristo, C., Iervolino, M., Vacca, A., 2012b. Discussion of "Analysis of dynamic wave model for unsteady flow in an open channel". J. Hydr. Engrg. ASCE, 138, 915-917.

Di Cristo, C., Iervolino, M., Vacca, A., 2015. On the stability of gradually varying mudflows in open channels. Meccanica, 50, 4, 963-979.

Dracos, T.A., Glenne, B., 1967. Stability criteria for openchannel flow. J. Hydraul. Div., 93(HY6), 79-101.
Gottlieb, S., Shu, C.W., 1998. Total variation diminishing Runge-Kutta schemes. Math. Comp., 67, 221, 73-85.

Harten, A., 1983. High resolution schemes for hyperbolic conservation laws. J. Comput. Phys. 49, 3, 357-393.

Huang, Z., Lee, J.J., 2015. Modeling the Spatial Evolution of Roll Waves with Diffusive Saint Venant Equations. J. Hydraul. Eng., 141, 2, 06014022-1.

Kenyon, K.E., 1998. Roll wave theory. Phys. Essays, 11, 531-540.

Kevorkian, J., Yu, J., Wang, L., 1995. Weakly nonlinear waves for a class of linearly unstable hyperbolic conservation laws with source terms. SIAM Journal on Applied Mathematics, $55,2,446-484$.

Khatsuria R.M., 2004. Hydraulics of Spillways and Energy Dissipators. CRC Press.

Kranenburg, C., 1990. On the stability of gradually varying flow in wide open channels. J. Hydraul. Res., 28, 5, 621628, doi:10.1080/00221689009499050.

Kranenburg, C., 1992. On the evolution of roll waves. J. Fluid Mech., 245, 249-261.

Liggett, J.A., 1975. Stability. Unsteady flow in open channel. In: Mahmood, K., Yevjevich, V. (Eds.): Water Resources Publications, Vol. 1. Englewood, Colorado.

Liu, K.F., Mei, C.C., 1989. Slow spreading of a sheet of Bingham fluid on an inclined plane. J. Fluid Mech., 207, 505-529.

Liu, Q.Q., Chen, L., Li, J.C., Singh, V.P., 2005. Roll waves in overland flow. J. Hydrol. Eng., 10, 2, 110-117.

Logan, M., Iverson, R.M., 2007. Video documentation of experiments at the USGS debris-flow flume 1992-2006 (amended to include 2007-2013). US Geological Survey, 2007-1315.

Montuori, C., 1963. Discussion of stability aspect of flow in open channels. J. Hydraul. Div., 894, 264-273.

Murdock, J.A., 1999. Perturbations: Theory and Methods. Classic in Applied Mathematics, SIAM.

Ponce, V.M., Simon, D.B., 1977. Shallow water propagation in open channel flow. J. Hydraul. Div., 103, 1461-1476.

Ridolfi, L., Porporato, A., Revelli R., 2006. Green's function of the linearized de Saint-Venant equations. J. Eng. Mech., 132, 2, 125-132.

Roe, P.L., 1986. Characteristic-based schemes for the Euler equations. Ann. Rev. Fluid Mech., 18, 337-365, doi:10.1146/annurev.fl.18.010186.002005

Stoker, J.J., 1957. Water Waves, the Mathematical Theory with Applications. Interscience, London.

Supino, G., 1960. Sopra le onde di traslazione nei canali. [Bore waves in channels]. Rend. Accad. Naz. Lincei, 295, 6, 543552. (In Italian.)

Thual, O., Plumerault, L.R., Astruc, D., 2010. Linear stability of the 1D Saint-Venant equations and drag parameterizations. J. Hydraul. Res., 48, 3, 348-353.

Witham, G.B., 1974. Linear and Nonlinear Waves. John Wiley \& Sons Interscience, New York.

Venutelli M., 2011. Analysis of dynamic wave model for unsteady flow in an open channel. J. Hydr. Engrg. ASCE, 137, 9, 1072-1078.

Zanuttigh, B., Lamberti, A. 2002. Roll waves simulation using shallow water equations and weighted average flux method. J. Hydraul. Res., 405, 610-622.

Zoppou, C., Roberts, S., 2003. Explicit schemes for dam-break simulations. J. Hydr. Engrg. ASCE, 129, 1, 11-34.

Received 19 June 2015 Accepted 16 October 2015 